International Journal of Addiction Research and Therapy
(ISSN:2637-8795)

\title{
Anger, Anxiety and Health Determinants in the Process of Community Recovery
}

\section{Pascal Scoles, DSW, LCSW}

Professor, Behavioral Health and Human Services and Director, Office of Collegiate Recovery, Student Life, Community College of Philadelphia, PA

\section{ABSTRACT}

The article discusses anger and anxiety in the context of recovery. It relates social determinants of health to individual pathology

*Correspondence to Author: and how an individuals stress level is related to one's community. Pascal Scoles, DSW, LCSW How stressors such as, drug infected areas, lack of access to Professor, Behavioral Health and parks and playgrounds, quality education, etc impact significantly on a person's anxiety is discussed. To help facilitate healthy change the use of peer specialists and partnering with the faithbased community can create a more comprehensive network of supportive allies.

How to cite this article:

Pascal Scoles.Anger, Anxiety and Health Determinants in the Process of Community Recovery. International Journal of Addiction Research and Therapy, 2020, 3:19

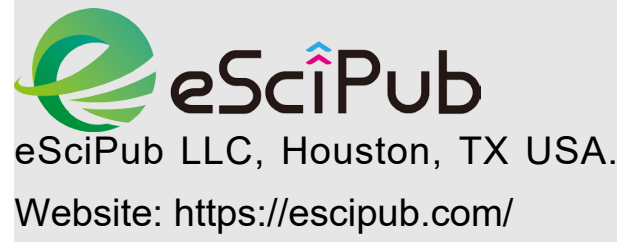


The etiology of anxiety and stress may be related to a person's bio-genetic structure, but how it gets expressed is often the result of psychosocial, environmental, and cultural factors. The degree to which these factors interact with genetics in the overall health of an individual has been a subject of debate among clinicians and researchers ${ }^{1}$. What is known is that deterioration of social determinants of health in neighborhoods is a predictive factor in a person's chances for recovery from addiction, trauma, and other behavioral health challenges ${ }^{2}$. If a community is dysfunctional and lacks necessary support for its members' economic and social sustainability, that individual's health and wellness have a high probability of declining. Community members living in high-risk areas where poverty and violence are considered a daily experience will just as likely create communal anxiety and stress ${ }^{3}$. From a holistic perspective, individual recovery wellness, not only refers to the person or the family but also must encapsulate the community 4 .

The successful path to recovery is very much restricted by the ability of a person to manage his/her anger, despair, and anxiety. Disruptions in the flow of one's recovery relapes occur when the body, due to stress, depression, anger, etc., is challenged by its inability to align their emotions and cognitive processes with their day to day reality. A positive flow of recovery is difficult to achieve when one's body, mind, and community are not in sync ${ }^{5}$. A person's lack of harmony is a major high-risk factor for recurring relapses. Clinical practitioners have been moderately successful in the treatment of specific disorders body and mind, but most often neglectful of the interplay between one's "individual pathology" and the "community pathology." The recognition that an individual's health is connected to a community's overall health is the missing link to consistent and efficient intervention strategies ${ }^{6}$. Without practitioners engaging in a comprehensive evaluation of the social determinants of health $\mathrm{SDoH}$, a successful long-term solution to the individual's behavioral health challenges can be nearly impossible. Incumbent upon the field of behavioral health is the obligation, not only to influence an individual's therapeutic choices toward making healthy lifestyle changes but also to work therapeutically in their communities to remove barriers to health access. Professionals must simultaneously help shape the community's perspective of what changes need to occur within and among their existing micro and meso systems to foster more positive and healthy lifestyle changes for all residents. This disequilibrium, for many, has led to a state of hopelessness and despair partly due to adverse health determinants such as, poorly nourished, or inadequate housing, etc. This body/mind dysphoria is almost always related to poor drug-related coping strategies. If there is a universal truth about addictive lifestyle individuals, it is that:

>1. They make poor appraisals of problems.

2. They are inflexible and have little creative, alternative plans and

3. They have no appreciation for the longterm effects of their actions.

Anger or hostility, although a typical human response, can inhibit one from reaching their goals or objectives. There are several conclusions researchers agree on concerning a person's anger:

$>$ *Anger is often a result of our desire to get what we want from someone.

$>{ }^{*}$ Anger is usually expressed to someone we love or toward friends because they fail to live up to our desires or wishes.

$>$ *The expression of anger is almost always interpreted as harmful and not helpful.

$>{ }^{*}$ The satisfaction after expressing anger does not achieve our goals in the long run 7 .

\section{Assessing anger and anxiety}

Coping with anger and anxiety in everyday life and managing stress without the use of alcohol and other drugs is a necessity if one is ever to redirect their life process and find well-being. Understanding the difference between aggression and assertiveness is an essential beginning to disarming anger. Aggression involves taking advantage of others by standing up for our rights in 
a hostile manner. Aggressiveness is generally viewed as an individual overpowers, humiliates, degrades, or belittles another individual. Aggressive individuals are more concerned about their rights, needs, and desires with no appreciation for the other person. Generally, aggression alienates us from others and turns us into suspicious, fearful, hostile individuals. On the other hand, assertive people stand up for their rights by direct, honest, and appropriate expression of their thoughts and feelings. They courteously relate to others, being sensitive to their demands but do not allow people to take advantage of them. An assertive person is flexible and understands he/she will not always get everything they want. In many ways, a confident person demonstrates many of the skills of an effective helper. They are empathic, they own their feelings, and they act responsibly. In a Jungian sense, they demonstrate the compensatory function. If the recovering person can operate impartially, he/she will demonstrate self-respect. ${ }^{8}$

Anger is sometimes referred to as anxiety turned inward. Many of the techniques to help control anxiety are like those used to manage anger. Stress is usually a result of emotional states of tenseness and distress that, at times, can result in violence. When inappropriate anger turns inside, the body reacts by heart palpitations, shortness of breath, loss of appetite, rapid breathing, sweating, trouble speaking, restlessness, etc. One's thoughts become confused, the individual has difficulty concentrating, and one has a fear of losing control, and an individual becomes selfconscious, hypervigilant, and memory function is impaired. One's sense of well-being is depressed, tense, jumpy, impatient, alarmed, edgy, etc. ${ }^{9}$. Although anxiety is also related to fear, a sense of vulnerability or helplessness leaves the person unable to cope with stressful situations without resorting to alcohol or drugs. The more that prejudice, errors, fantasies influence consciousness, and infantile wishes, the more the already existing dissociative gap will widen into a neurotic derealization and lead to artificial life, far removed from healthy instincts, nature, and truth ${ }^{10}$. Change in one's emotional experience appears to follow a series of stages, which is more spiral or circular in progression than linear. Most people in early recovery progress from sobriety to relapse and back to sobriety, with the sequence related to guilt, embarrassment, and shame regarding regression to earlier stages of addiction ${ }^{11}$.

\section{Determinants of Health in Recovery}

Studies have found that increases in income, educational opportunities, and accessible housing have the most significant positive effect on the development of healthy lifestyles ${ }^{12}$ and that social spending, not health care spending, is significantly associated with improvement in mortality rates ${ }^{13}$. Research indicates a high correlation among these social inequalities, which refer to differences in the health of individuals or groups and health disparities; thus, confounds our ability to improve the health of a community 2. Studies have found that increases in income, educational opportunities, and accessible housing have the most significant positive public health effect on the individual ${ }^{1213}$. Eighty percent $80 \%$ of individuals' health is determined by their behaviors and the social and environmental conditions in which they work, live, and play ${ }^{14}$. Partly based on a result of these findings, the behavioral health field is currently engaged in a movement to expand the traditional medical/psychiatric medication driven model of care with a more comprehensive and inclusive perspective on health care ${ }^{16}$. This health care goal is to redirect and focus on the social determinants that impact a person's health and reduce health inequities and disparities among different population groups. From an addiction recovery perspective, a population health focus is defined by a clinician's ability to counsel individuals and their families while simultaneously being a community interventionist to influence complex social, behavioral, and environmental factors affecting the recovery populations within a given neighborhood. This therapeutic shift to a comprehensive, holistic model of healing is a realization that the traditional methods of behavioral health care delivery are not expansive enough to 
effectively impact the drug and alcohol concerns for the individual, family, or community. This health care model is a shift from a professionallydriven approach to a system of care that provides lifetime support while recognizing the many pathways to health ${ }^{15}$. In many ways, "Where we live, work, learn, and play is as significant as our genetic code" 2 . The current behavioral health system, which focuses on specific acute disorders, continues to be inadequate in helping communities surrounding outpatient and inpatient services to develop healthy lifestyles. Thus, professionals in varied disciplines, education, psychology, social work, nursing, etc., are seeing more significant evidence that a person's health cannot be separated from an individual's community health ${ }^{6}$

Moreover, many professionals and recovery advocates believe a lack of attention to these social determinants contributes to the overall "community pathology" and low rates of therapeutic success ${ }^{16}$. Therapeutic healing catalysts are found in addressing those social determinants that influence many lifestyle choices. Thus, from a community health perspective, healing the community heals the individual, understanding that one inherently does not exist without the other.

Environmental and social exposure to factors such as high crime and drug-infested areas, domestic violence, and lack of access to parks or playgrounds, transportation, quality education, social services, and mental health care have a significant impact on lifestyle choices. Therefore, from a holistic behavioral health perspective, it would best be defined by an advocacy impact on a person's anxiety and stress to intervene and influence these complex negative social, behavioral, and environmental factors. These advocacy impacts can be accomplished by actively working to engage, community organizations, families, schools, and individuals to create and shape positive and healthy environments in which all members can thrive ${ }^{17}$. Indeed, behavioral health challenges exist that require diagnosis and medical and psychosocial interventions. Surely, to survive in the behavioral health field, clinicians must know and contribute to the diagnostically driven payment system of healthcare. The DSM does accomplish its objective of providing an understanding of some of the complex biopsychosocial concepts of psychiatric diagnosis. Its weakness is not so much in its attention to individuals and families but its neglect of the influence of a community's impact on a person's stressful life ${ }^{5}$.

Partly due to the medical focus on payment, most of the history of behavioral health care, community involvement, and neighborhood connection were viewed as something that happened near the end of treatment, depending on adherence and symptom remission and control 18. Systems did not see the community resource for promoting people's health, but as a place to which people might be released when they were "healthier." Before pursuing any workforce activity or educational studies, people were instructed to wait until they had achieved abstinence or sobriety. The treatment community simply did not view neighborhood anxiety in which individuals lived as a possible contributing factor to either the problem or the solution. Also, the person receiving services and their supporters were not considered a significant participant in these decisions regarding their etiology or treatment/recovery plan. All too often, a person's family, key allies, spiritual resources were seldom invited into an individual's planning process. Community connections were considered the purview of non-medical staff-and even then, were done as referrals rather than intentional primary health resources ${ }^{18}$.

Historically, the lack of a comprehensive vision of health that focused on the influencing social determinants of health SDoH is traced partly to the helping professions. The cultural and ethnic sensitivity to community processes involved in determining health and pathology in one's community were factors that were lacking in traditional healthcare ${ }^{16}$.

To offset this historical bias and effectively support individuals and their communities a coalition of different healing professionals are attempting 
to manage and influence the recovery process by ${ }^{1}$ building health environments through access to better primary health care and health literacy, ${ }^{2}$ building economic stability by partnering with agencies to providing employment, food security, housing stability, and workforce activities, and ${ }^{3}$ supporting educational access to language and literacy, early childhood education and development, enrollment in college or trade schools, support social cohesion, etc. ${ }^{16}$. A transformed health care system must embrace the concept that "the health of individuals is affected by the health of the overall community. Provider agencies exist within the community. They are members of a given community and have a responsibility to participate in improving the overall health of the community" 18 .

\section{Certified Peer Specialist}

At the heart of a healthy community system of care is the belief that people living with behavioral health challenges can recover. People with lived experience can provide vital support to each other in achieving long-term recovery. They can also play essential roles in designing, delivering, and evaluating professional therapeutic services. A growing number of behavioral health programs and organizations are mobilizing peer recovery support personnel through the development of healthy peer cultures and assertively linking people to local communities of recovery. A kind of case manager, the certified peer specialist, is seen as a person with expertise based on training and life experience. The peer specialist and provider of service form a relationship to recognize and respect the unique contribution that each brings to the therapeutic process and who work together to develop approaches, solutions, and treatment and recovery plans with the person receiving services. ${ }^{19}$ Peer specialist culture's evolution recognizes that everyone in the "community" has knowledge and skills that can be used to solve whatever challenges they face together. The person receiving services, as well as the service provider, is viewed as an essential and helpful resource. In a peer culture, people in recovery should be employed at all levels of the system. Through alumni organizations, providing leadership opportunities on agency boards and committees, enlisting the knowledge and resources of the community of people in recovery, their families, and other supporters, they facilitate development and linkages with peer support groups within the agency in the city ${ }^{19}$.

\section{Faith Community}

Medical/psychiatric care and peer leadership are just two of the many formal and informal community resources that contribute to building a healthier recovery community. Spirituality is a third and valuable addition to managing life's stress and anxiety. Spirituality means different things to different people; most agree that experiencing and appreciating the sacred within or beyond our material world is reflective of this idea. Spirituality is another dimension of human existence beyond the biopsychosocial framework used to express resilience and transformation. Social service educators, practitioners, and individuals receiving services need to expand their frame to include a religious/spiritual dimension ${ }^{20}$. Some clinicians have taken on what Bloch ${ }^{21}$ calls "a consumerist and personal approach to the acquisition of religious or spiritualized information" (p. 9). Bloch maintained that such persons seek a variety of spiritual experiences based on "a desire to reduce social uncertainty by seeking compatibility across different knowledge claims, rather than having to select one at the absolute social cost of another" (p. 9). Thus, it is not uncommon for contemporary spiritualists to embrace multiple belief systems that may include some different theological material. Generally, religion is a more structured belief system involving emotion, morality, and a sense of identity and a specific understanding of the community. Affiliation with a faith-based institution improves the physical, social, and emotional health of individuals ${ }^{22}$ Ungar and his colleagues identified seven aspects of resilience across many different cultures. Each depends on the other. For example, attending an organized, structured place of worship has been shown to 
increase a child's social networking skills, provide a feeling of cohesion and belonging in a person's community. The above process promotes a sense of personal control and a protective sense of social justice when threatened by life events. It is this complexity and multilevel nature of resilience that explains how people use the internal and external resources assets that are both available and accessible in the faith-based communities to overcome adversity ${ }^{23}$.

Dunlap 2008 believes that a person's difficulty embracing traditional faith-based institutions and spirituality has to do with a prejudicial thought that even today, people of faith discriminate against science and fear that emotion and science could coexist ${ }^{24}$. Historically, the coexistent fellowship of religion and science was very much a part of the self-help movement. Research on people in recovery has found statistically significant levels of faith and spirituality than those continuing to relapse; also, relapsing individuals show significantly lower religiosity levels than those in recovery 25 .

We know that building healthy communities must include faith ministries and other support groups who are providing strength and hope to people with behavioral health challenges. We also know that behavioral health providers have witnessed the power of religion and spirituality as an essential tool for healing. In a comprehensive behavioral health management approach, we must embrace a strategy geared toward the elimination of stress in the overall community; be attentive toward environmental factors such as divorce, death, and illness; and support and provide opportunities for better housing, increased employment opportunities, and positive family activities.

The faith community realizes that without attention to these communal interventions, one will continue to live in a static environment or a neighborhood in decline, which becomes a toxic wasteland for individuals, their families, and their community. The general bias to integrating the faith-based community into the mainstream of the treatment community reflects, to some extent, the fear and distrust traditional counselor education and training have regarding how others interpret and talk about reality ${ }^{26}$.

Recovery support groups do not share this fear and mistrust by some of the helping professions. They outline a way of living that is not just related to issues of chemical use and abuse but provides a spiritual path to a more positive life worth living. For many, the Steps are more than just a flow about recovery but a life of meaning and transcendence. As stated in the original tenants of the Oxford movement, the flow to a successful, positive experience depends on one's ability to:

-examine conscience,

-review our defects,

-make restitution to people we hurt, -resolve to help others in need, and

-find a spiritual space to practice our newfound wisdom.

Although these five tenants were developed from a Christian perspective, they are positive principles reflected in all faith-based organizations ${ }^{27}$.

Contrary to some beliefs, people are very good at determining what will increase positive emotions that support a positive flow between spirit and matter. Many techniques have developed to help improve one's positive outlook on life ${ }^{28}$. All successful counseling strategies share with the faith community, the following behavioral path to healing: ${ }^{1}$ Changing your activities, ${ }^{2}$ Changing your thinking, ${ }^{3}$ Nurturing relationships, ${ }^{4}$ Valuing personal growth, and ${ }^{5}$ Decreasing negative emotions ${ }^{29}$.

\section{Conclusion}

The management of anger and anxiety is often the result of a complex interaction between psychosocial, environmental, and cultural factors. These factors that facilitate anxiety and stress can best be managed by intervening upon and influencing social, behavioral, and environmental factors by working to engage individuals, their families, schools, and community organizations. Through this engagement, people can create 
and shape positive and healthy environments in which all members can thrive. Studies have found that increases in income, educational opportunities, and accessible housing have the most significant positive effect on building healthy lifestyles. To create a more comprehensive health care network of providers, one needs to embrace more fully the evolving peer specialist movement and expand our partnership with the faith community.

\section{References}

1. Way, B. and D. Lieberman, (2010). Is there a genetic contribution to cultural differences? Collectivism, individualism, and genetic markers of social sensitivity. Social Cognitive and Affective Neuroscience, Volume 5, Issue 2-3, June/September, Pages 203-211.

2. Michener, J., D. Koo, B. Castrucci, and J. Sprague, (Eds). Practical Playbook, Public Health, and Primary Care Together. (2016). Oxford University Press.

3. Death by ZIP code: Investigating the Root Causes of Health Inequity. (2016). AMA.org.

4. Scoles, P. (2020). Building recovery resilience through culture, community, and spirituality. Journal of Behavioral Health. Volume 9.

5. Scoles, P. (2020). Substance Abuse Treatment in Recovery. Kindle Publication.

6. Scoles, P. (2020). Trauma, Addiction, and the Community. Sober World. Vol. 9. Issue 1.

7. Kleinke, C. (1994). Common Principles of Psychotherapy. California: Brooks/Cole.

8. Egan, G. (2015). The Skilled Helper a Problemmanagement and Opportunity-development Approach to Helping. Canada: Crane Library.

9. Siegel, B. S. (1989). Peace, love \& healing. New York: Harper \& Row.

10. Jung, C (1964). Man and His Symbols. New York: Anchor Books.

11. Scoles, P. (2019). The Flow of Recovery. Counselor. Vol. 20, No. 1, pp. 33-37.

12. Frieden TR. A framework for public health action: the health impact pyramid. Am J Public Health. 2010;100(4):590-595.

DOI:10.2105/AJPH.2009.185652

13. Healthy People 2000 Progress Review. (1997). U.S. Department of Health and Human Services. Rockville, MD: Alcohol, Drug Abuse, and Mental Health Administration.

14. Magnan, S., 2017. Social Determinants of Health 101 for Health Care: Five Plus Five. NAM Perspectives. Discussion Paper, National Academy of Medicine, Washington, DC. DOI: 10.31478/201710c

IJART:https://escipub.com/international-journal-of-addiction-research-and-therapy/
15. Lamb, R., Evans, A. C., \& White, W. L. (2009). The role of partnership in recovery-oriented systems of care: The Philadelphia experience. Retrieved from www.facesandvoicesofrecovery.org

16. Scoles, $P$, and F. DiRosa (2018). Social Determinants of Health and Behavioral Health Challenges. Counselor. 19/3. May/June. pp. 28-31.

17. Levine, C., and T. Murray. (2004). The Cultures of Caregiving: Conflict and Common Ground among Families, Health Professionals, and Policy Makers. Johns Hopkins University Press.

18. http://dbhids.org/2015/01/Practice-Guidelines

19. Tools for Transformation Series: Peer Culture/Peer Support/Peer Leadership. Philadelphia Department of Behavioral Health and Intellectual disAbility Services (2006). Arthur C. Evans, Ph.D., DBHIDS Commissioner, City of Philadelphia.

20. Grim, B.J., Grim, M.E. (2019). Belief, Behavior, and Belonging: How Faith is Indispensable in Preventing and Recovering from Substance Abuse. Journal of Religious Health. Vol.58, 1713-1750.

21. Bloch, J. (1998). How we Think they Think: Anthropological approaches to cognition, memory, and literacy. Boulder $\mathrm{CO}$ and Oxford: Westview Press, 205 pp., £37.95, ISBN 081333373 3. Africa, $\quad 71(3), \quad 519-520$. DOI:10.3366/afr.2001.71.3.519

22. Parrott, L., Plante, T. G., Sherman, A. C. (2001). Faith and Health: Psychological Perspectives. United Kingdom: Guilford Publications

23. Ungar, M. (2004). Change Your World: The Science of Resilience and the True Path to Success Kindle Edition.

24. Dunlop, P. (2008). Awakening our Faith in the Future: The Advent of Psychological Liberalism. Kindle Publications

25. Jarusiewicz, B. (2000) Spirituality, and Addiction, Alcoholism Treatment Quarterly, 18:4, 99-109, DOI: 10.1300/J020v18n04_08.

26. Scoles P. (2020) Spirituality, Culture, and the Process of Assessment in Recovery. J Addictive Behaviors, Therapy \& Rehabilitation. Vol. 9:2

27. Alcoholics Anonymous Comes of Age: A Brief History of A.A. (1985). New York: Alcoholics Anonymous World Services.

28. Gaithersburg, MD. (2019). Assessing Health Outcomes in a Community Development Context: Lessons Learned from the Evaluation of the Success Measures Health Tools. Community Science.

29. Fordyce, M. W. (1983). A program to increase happiness: Further studies. Journal of Counseling Psychology, 30(4), 483-498. https://doi.org/10.1037/0022-0167.30.4.483 